\title{
Colorectal cancer pathology reporting: a regional audit
}

\author{
A D Bull, A H B Biffin, J Mella, A G Radcliffe, J D Stamatakis, R J C Steele, G T Williams
}

Department of Pathology, University of Wales College of Medicine, Cardiff A D Bull

G T Williams

Clinical Effectiveness Support Unit (Wales), Penarth

A H B Biffin

Department of Surgery, University of Nottingham,

Nottingham

J Mella

R J C Steele

Department of Surgery, Llandough Hospital and Community NHS Trust, Penarth A G Radcliffe

Department of Surgery, Bridgend and District NHS Trust,

Bridgend

J D Stamatakis

Correspondence to: Professor G T Williams, Department of Pathology, University of Wales College of Medicine, Heath Park, Cardiff CF4 4XN.

Accepted for publication 5 November 1996

\begin{abstract}
Aims-To audit the information content of pathology reports of colorectal cancer specimens in one National Health Service region.

Methods-All reports of colorectal cancer resection specimens from the 17 NHS histopathology laboratories in Wales during 1993 were evaluated against: (a) standards previously agreed as desirable by pathologists in Wales; and (b) standards considered to be the minimum required for informed patient management.

Results-1242 reports were audited. There was notable variation in the performance of different laboratories and in the completeness of reporting of individual items of information. While many items were generally well reported, only $51.5 \%$ (640/ 1242) of rectal cancer reports contained a statement on the completeness of excision at the circumferential resection margin and only $30 \%(373 / 1242)$ of all reports stated the number of involved lymph nodes. All of the previously agreed items were contained in only $11.3 \%(140 / 1242)$ of reports on colonic tumours and $4.0 \%$ (40/1242) of reports on rectal tumours. Seventy eight per cent $(969 / 1242)$ of colonic carcinoma reports and $46.6 \%$ (579/ 1242) of rectal carcinoma reports met the minimum standards.

Conclusions-The informational content of many routine pathology reports on colorectal cancer resection specimens is inadequate for quality patient management, for ensuring a clinically effective cancer service through audit, and for cancer registration. Template proforma reporting using nationally agreed standards is recommended as a remedy for this, along with improved education, review of laboratory practices in the light of current knowledge, and further motivation of pathologists through their involvement in multidisciplinary cancer management teams.

(f Clin Pathol 1997;50:138-142)
\end{abstract}

Keywords: colorectal cancer; histopathology; audit quality; guidelines; protocols.

Histopathological reporting of resection specimens for colorectal cancer provides important information both for the clinical management of the affected patient and for the evaluation of health care as a whole. For the individual patient, it confirms the diagnosis and describes variables that affect prognosis, ${ }^{1}$ notably the extent of the disease (the pathological stage) and the completeness of local excision, both of which inform future clinical management. For example, a number of randomised trials have demonstrated the benefit of adjuvant chemotherapy for tumours that have spread to the regional lymph nodes (Dukes' C cases). ${ }^{2}$ In rectal cancer, there is evidence that involvement of the circumferential resection margin is an important predictor of local recurrence and patient survival ${ }^{3}$ which might identify patients who would benefit from postoperative adjuvant therapy. ${ }^{4}$ In addition, pathology reports are often used by insurance companies in assessing financial risk when patients seek insurance after a diagnosis of cancer. For health care evaluation, pathology reports provide potentially robust information for cancer registration, for clinical audit, for assessing the accuracy of new diagnostic and preoperative staging techniques, and for ensuring comparability of patient groups in clinical trials. It is crucial, therefore, that pathology reports of colorectal cancer specimens contain the information that is necessary to fulfil these functions, and that this information is accurate and complete.

Guidelines on the information content of pathology reports in colorectal cancer have been published in standard textbooks of pathology ${ }^{5}$ and by various expert working groups. ${ }^{7-10}$ The recommendations have varied in detail and complexity, ranging from comprehensive lists of data items that encompass all variables that could be of conceivable relevance to prognosis to more focused, pragmatic 'minimum requirements' whose relevance to current patient management attempts to be more evidence-based. However, there is no published information on the quality of colorectal cancer reporting in current histopathology practice in the UK. In this paper, we present the findings of an audit of pathology reports of colorectal cancer specimens in all of the NHS histopathology laboratories in one UK region (Wales) during 1993, a study that was undertaken as part of a wide ranging audit of all aspects of colorectal cancer management in the Principality and in the Trent region of England whose initial findings have been presented recently. ${ }^{11}$

\section{Methods}

The population based audit of colorectal cancer management in Wales was directed by an expert working party set up by the Royal College of Surgeons of England; the methods 
Table 1 Data items abstracted from pathology reports and used for audit

Colonic carcinomas
Length of the specimen
Size of the tumour (maximum dimension)
Distance of tumour from nearest resection end
Macroscopic description of the appearance of the tumour
Histological type of the tumour
Histological grade of the tumour
Extent of tumour invasion into and beyond the bowel wall
Involvement of the resection ends by tumour
Involvement of regional lymph nodes by tumour
Number of lymph nodes involved
Dukes' stage
Rectal carcinomas
All of the above plus:
Involvement of the circumferential excision plane
Minimum distance of clearance at the circumferential
excision plane

Table 2 Minimum criteria for an adequate report

Colonic carcinomas

Histological type of the tumour

Histological grade of the tumour

Extent of tumour invasion into and beyond the bowel wall

Involvement of the resection ends by tumour

Involvement of regional lymph nodes by tumour Rectal carcinomas

All of the above plus:

Involvement of the circumferential excision plane

used will be described in detail elsewhere. Briefly, all patients with colorectal cancer treated in the 17 NHS district general hospitals in Wales during the calendar year of 1993 were identified from a comprehensive search of a number of data recording systems and, after obtaining permission from the surgeons and histopathologists involved, copies of the pathology reports were obtained by data collectors. The presence or absence of a statement on items of information in the pathology reports (11 for colonic cancers, 13 for rectal cancers) was recorded on a proforma by a single surgical research fellow (JM), and transferred to a computer database by an optical mark scanner to be analysed using the Statistical Package for Social Sciences (SPSS for Windows, SPSS Inc., Chicago, Illinois, USA). Data recording was validated by checking the abstracted information from a randomly selected $10 \%$ of reports against the original pathology report by one of us (ADB).

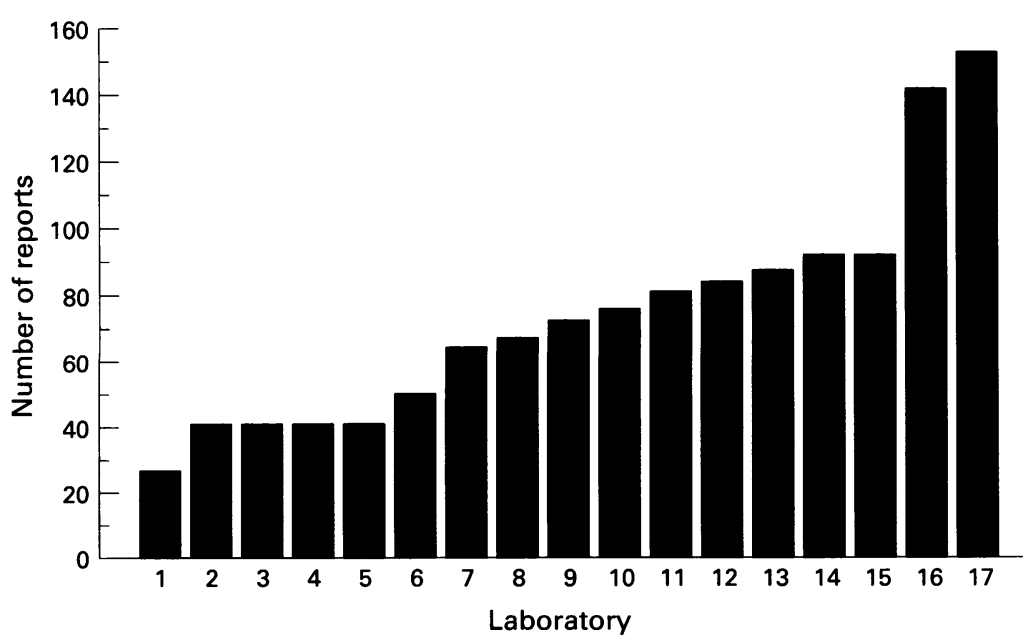

Figure 1 Histogram showing the number of pathology reports obtained from each of the 17 laboratories in Wales.
The items of pathology information recorded are shown in table 1 . They were based on standards for pathology reporting of colorectal cancer that had been agreed previously by histopathologists in Wales in March 1992 after discussion of published guidelines from the literature at regional pathology audit meetings. Pathologists from 15 of the 17 Welsh laboratories had attended at least one of the two meetings at which the standards were set, and all histopathologists in Wales were subsequently circulated with the agreed conclusions.

The informational content of all of the pathology reports was audited against the agreed standards, both for Wales as a whole and for each of the 17 laboratories. The percentage of reports containing a statement on each of the data items listed in table 1 was obtained, and also the percentage containing statements on all of the data items (11 for colonic tumours, 13 for rectal tumours). Finally, the percentage of reports containing statements on all of the data items in table 2 was obtained, these less rigid criteria being considered by us to be the minimum necessary for an adequate report. During the study, the identity of the 17 laboratories was known only to one of the investigators (AHBB, an independent data manager) who, after the audit was completed, provided each of the laboratories with a report of its own performance, together with that of each of the other laboratories (anonymised) and of the aggregated Welsh laboratories as a whole.

\section{Results}

In total, 1242 pathology reports were available for assessment, $57.2 \%$ of which were resections for colonic cancer and $42.8 \%$ for rectal cancer. The reports were issued from 17 NHS laboratories staffed by 36 consultant histopathologists. Although pathologists professing a special interest in gastrointestinal disease worked at some laboratories, the responsibility for reporting colorectal cancer specimens was always shared between all department consultants. Figure 1 shows the distribution of the number of reports obtained from each laboratory, which varied from 27 to 152 (median 72).

Validation of the surgical research fellow's abstracted data by a pathologist in a random sample of $10 \%$ of the reports showed agreement with the interpretation of the original report for the vast majority of items of information recorded. Virtually all of the discrepancies related to statements which were not completely clear in the original reports; in such cases, the surgical research fellow had nearly always interpreted the report generously and had recorded the data item as being present.

Table 3 shows the percentage of reports containing statements on each of the individual items of information defined by the standards in table 1 . It can be seen that performance varied considerably for different data items and between laboratories. All reports described the histological type and grade of the tumour and although almost all stated the extent of invasion into or beyond the bowel wall, this information 
Table 3 Percentage of reports containing statements on individual data items audited

\begin{tabular}{|c|c|c|c|c|}
\hline Data item & $\begin{array}{l}\text { Worst performing } \\
\text { laboratory }\end{array}$ & $\begin{array}{l}\text { Best performing } \\
\text { laboratory }\end{array}$ & $\begin{array}{l}\text { Median for } 17 \\
\text { laboratories }\end{array}$ & $\begin{array}{l}\text { Value for Wales as } \\
\text { a whole }\end{array}$ \\
\hline \multicolumn{5}{|l|}{ For all tumours: } \\
\hline Length of specimen & 84.1 & 100.0 & 98.4 & 96.7 \\
\hline Tumour size & 75.0 & 98.7 & 94.4 & 92.5 \\
\hline Distance from resection end & 52.5 & 82.9 & 75.2 & 72.9 \\
\hline Appearance of tumour & 36.1 & 98.4 & 92.0 & 84.9 \\
\hline Histological type & 100.0 & 100.0 & 100.0 & 100.0 \\
\hline Histological grade & 100.0 & 100.0 & 100.0 & 100.0 \\
\hline Extent of invasion & 77.8 & 100.0 & 98.6 & 96.7 \\
\hline Resection end involvement & 55.1 & 100.0 & 92.3 & 91.0 \\
\hline Whether nodes involved & 85.0 & 100.0 & 95.3 & 95.2 \\
\hline Number of nodes involved & 14.4 & 48.9 & 27.5 & 30.0 \\
\hline Dukes' stage & 12.5 & 93.8 & 73.6 & 74.9 \\
\hline \multicolumn{5}{|l|}{ For rectal tumours only: } \\
\hline Circumferential plane involvement & 8.3 & 78.1 & 57.6 & 51.6 \\
\hline Measured circumferential plane clearance & 0 & 28.1 & 7.7 & 12.1 \\
\hline
\end{tabular}

Table 4 Percentage of reports fulfilling standards

\begin{tabular}{|c|c|c|c|c|}
\hline \multirow[b]{2}{*}{ Laboratory } & \multicolumn{2}{|c|}{ Originally agreed standards (table 1) } & \multicolumn{2}{|c|}{ Minimum standards (table 2) } \\
\hline & Colonic tumours ${ }^{*}$ & Rectal tumours* & Colonic tun & Rectal tumours* \\
\hline A & $2.6(4)$ & $0(1)$ & $44.7(1)$ & $5.9(1)$ \\
\hline B & $7.1(6)$ & $0(1)$ & $50.0(2)$ & $8.3(2)$ \\
\hline C & $0(1)$ & $0(1)$ & $52.0(3)$ & $20.0(3)$ \\
\hline D & $9.4(9)$ & $0(1)$ & $85.0(10)$ & $28.8(4)$ \\
\hline E & $28.3(17)$ & $0(1)$ & $93.5(16)$ & $33.3(5)$ \\
\hline $\mathbf{F}$ & $5.1(5)$ & $8.6(15)$ & $82.1(9)$ & $40.0(6)$ \\
\hline G & $10.0(11)$ & $2.9(8)$ & $68.6(5)$ & $41.0(7)$ \\
\hline $\mathrm{H}$ & $0(1)$ & $5.9(12)$ & $95.8(17)$ & $41.2(8)$ \\
\hline I & $14.9(13)$ & $8.8(16)$ & $54.5(4)$ & $47.1(9)$ \\
\hline $\mathrm{J}$ & $0(1)$ & $0(1)$ & $80.0(8)$ & $50.0(10)$ \\
\hline $\mathrm{K}$ & $13.3(12)$ & $3.0(9)$ & $88.0(13)$ & $51.5(11)$ \\
\hline L & $16.2(14)$ & $4.7(11)$ & $74.5(7)$ & $58.1(12)$ \\
\hline $\mathbf{M}$ & $7.4(7)$ & $8.3(14)$ & $70.4(6)$ & $58.3(13)$ \\
\hline $\mathbf{N}$ & $9.8(10)$ & $3.2(10)$ & $87.5(12)$ & $64.5(14)$ \\
\hline $\mathrm{O}$ & $8.8(8)$ & $0(1)$ & $86.5(11)$ & $65.2(15)$ \\
\hline $\mathbf{P}$ & $19.4(15)$ & 7.1 (13) & $88.9(14)$ & $71.4(16)$ \\
\hline Q & $19.7(16)$ & $10.1(17)$ & $92.9(15)$ & 73.7 (17) \\
\hline All Wales & 11.3 & 4.0 & 78.1 & 46.6 \\
\hline
\end{tabular}

${ }^{\star}$ Figures in brackets represent performance ranking of laboratories: $1=$ worst; 17 = best.

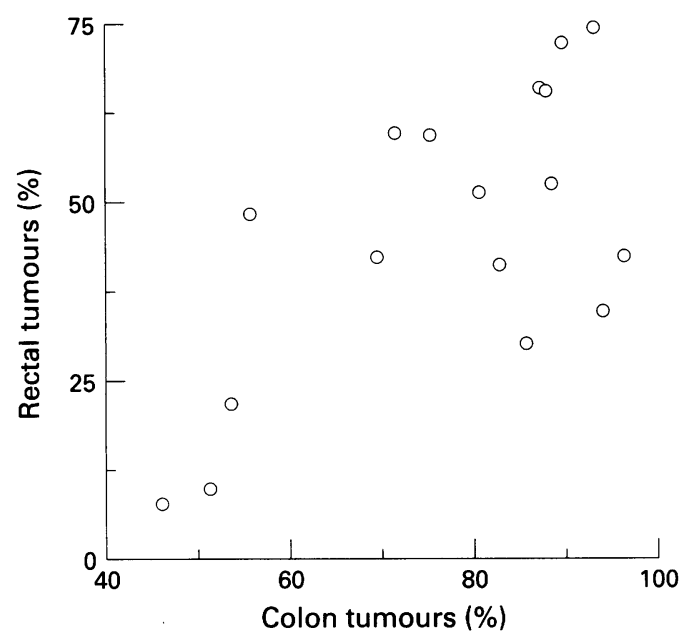

Figure 2 Regression plot of laboratories' performance (\% of cases fulfilling minimum standards) for reporting colonic carcinomas versus rectal carcinomas. There is a positive correlation (Spearman's rank correlation coefficient $\rho=+0.527, p<0.05)$.

was invariably present in the reports of only five laboratories. Whether or not the lymph nodes were involved was also generally well reported (95.2\% overall) but four laboratories failed to give this very important item of information in every case. However, the absolute number of lymph nodes involved was poorly reported, being given in only $30 \%$ of cases overall with no laboratory achieving a compliance of $>50 \%$. Formal Dukes' staging of the tumour was given in $74.9 \%$ of cases, varying notably between laboratories from 12.5 to $93.8 \%$.

A statement on completeness of excision at the ends of the specimen was given in $91 \%$ of reports, but only two laboratories achieved $100 \%$. The distance from the tumour to the nearest resection end was less frequently mentioned. However, completeness of excision at the circumferential excision plane in rectal carcinomas was poorly reported, being mentioned in only one half of all reports and the distance of clearance being measured in less than one in eight. Whereas the best performing laboratory gave these two data items in $78.1 \%$ and $28.1 \%$ of reports, respectively, seven laboratories failed to describe the circumferential plane in $>50 \%$ and four never mentioned the distance of clearance.

Table 4 shows the percentage of pathology reports containing statements on all of the data items shown in tables 1 and 2 for each of the laboratories and for Wales as a whole. The proportion of cases containing information on all of the items in table 1 represent those fulfilling all of the requirements originally agreed by the pathologists in Wales before the audit commenced. The results are disappointing with only $11.3 \%$ of colonic cancer reports and $4.0 \%$ of rectal cancer reports meeting all of the agreed standards. Reports from two laboratories never contained all of the required data items, and no laboratory fulfilled the standards in $>20 \%$ of colonic tumours and $>10 \%$ of rectal tumours.

The proportion of cases containing statements on all of the items in table 2 represent those fulfilling criteria considered by us to be the minimum for an adequate report. This standard was only achieved in $78.1 \%$ of colonic cancer reports and $46.6 \%$ of rectal cancer reports. No laboratory achieved $100 \%$ for either tumour, and only six of the 17 achieved both $>80 \%$ for colonic tumours and $>50 \%$ for rectal tumours. There was no significant correlation between laboratories' performance against the agreed guidelines and their performance against the minimum standards (Spearman's rank correlation). For example, laboratory $\mathrm{J}$ failed to satisfy the agreed criteria in any report but satisfied the minimum criteria in $80 \%$ of colonic cancer reports and $50 \%$ of rectal cancer reports. However, a significant correlation $(p<0.05)$ was found between 
laboratories' performance in reporting colonic and rectal cancers when the minimum standards only were assessed (fig 2, Spearman's rank correlation).

\section{Discussion}

Quality pathology reports of colorectal cancer specimens are central to ensuring clinical effectiveness in the management of this common malignancy. Inadequate histopathology can lead to inappropriate therapy and misleading information for patients. It is, therefore, of considerable concern that this audit of pathology reporting in Wales has highlighted important deficiencies in about one fifth of reports on colonic cancer resections and in half of reports on rectal cancer resections. There is no reason to believe that the situation in Wales is any different from other health regions in the UK. The wide ranging clinical audit of colorectal cancer from which this study emerged also evaluated performance in the Trent region of England. ${ }^{11}$ Although pathology data were recorded for Trent, they are not available in a form that is strictly comparable with those from Wales but, nevertheless, a preliminary evaluation indicates that overall performance was not significantly different (unpublished observations). Therefore, it is appropriate to consider the nature of the Welsh audit in some detail.

It is very important to make clear that we audited only the information content of issued reports. We have not investigated diagnostic precision, the way that the specimens have been handled, the sampling of the specimens for microscopic examination, or the accuracy of any of the data. Although all of these factors will obviously influence the quality of the information that is presented in a pathology report, we have assessed only the completeness of the final report with regard to a defined number of items of information.

One strength of this audit is that it has encompassed all of the pathology reports that could be retrieved in one year from all 17 laboratories in the region, and should therefore be relatively free from selection bias. Another feature is that the information content of the pathology reports was abstracted for the audit process by an independent surgical research fellow. This meant that it was interpreted by an unbiased end-user of the report rather than by a pathologist who might be tempted to infer information when it was not clearly presented. In fact, validation of the abstracted data in a random sample of $10 \%$ by a pathologist identified few discrepancies, and most of these indicated a generous interpretation of equivocal items of information by the surgeon.

The findings of this audit suggest that the informational content of colorectal cancer reports issued by NHS pathology laboratories leaves much to be desired. Although it could be argued that some of the data items required are not essential for informed patient management, such as the length of the specimen, the size and appearance of the tumour, and the distance of the tumour from the nearest resection end, these are all items that would generally be regarded as reflecting careful examination of the specimen and would contribute to the value of the report in clinical auditing of preoperative patient assessment and surgical technique. Similarly, a statement of the pathological Dukes' stage might not be essential if the information required to derive this is contained within the report, but some concluding statement on pathological stage will greatly facilitate cancer registration as registries move towards recording this routinely. However, the most important deficiencies relate to the poor description of circumferential resection plane involvement in rectal carcinomas and the number of lymph nodes involved by metastases. There is now strong evidence that circumferential margin involvement in rectal cancer is an important prognostic indicator, having high predictive value for both survival and local recurrence. ${ }^{3} 12$ Accurate reporting of circumferential margin involvement is likely to have considerable influence on the decision whether to use adjuvant radiotherapy or chemotherapy. Our finding that this information was given in only a half of rectal cancer reports suggests that effective patient treatment may be being compromised by incomplete pathological reporting. Involvement of lymph nodes is another factor that is used to select patients for postoperative chemotherapy. ${ }^{2}$ Moreover, the actual number of lymph nodes involved also has independent prognostic significance, ${ }^{1}$ sufficient to warrant separation of cases with less than four positive nodes from other node-positive cases in both the TNM and the Jass staging systems. ${ }^{13}{ }^{14}$ It may be that the reluctance of pathologists to report the number of positive nodes in this audit is a reflection of the fact that this item does not contribute to the Dukes' classification, which is most widely used in the UK.

The frequent failure of reports to contain information on circumferential rectal margin involvement and the number of positive lymph nodes, coupled with the lack of a stated Dukes' stage and a comment on the distance from the tumour to the resected end of the specimen in about one quarter, are largely responsible for the fact that few reports met all of the standards originally agreed by the pathologists in Wales. Because of this, the reports were also audited against a minimum set of standards that were regarded as essential for postoperative management of the patient. Not surprisingly, this led to considerably improved results. Nevertheless, only $78.1 \%$ of colonic cancer reports and $46.6 \%$ of rectal cancer reports were complete. The difference between these two figures can be accounted for mainly by the poor reporting of circumferential margin involvement in rectal tumours, and this is clearly the most important factor requiring urgent remedial attention. Figure 2 shows that there was a correlation between individual laboratories' performance in reporting colonic and rectal tumours, indicating that there are some laboratories whose overall performance is clearly inferior to others. These differences may be related to variations in laboratory workloads and resources, but it is possible that 
the motivation of pathologists and their understanding of the importance of some of the items requiring description in the reports are also partly to blame. For example, appreciation of the prognostic value of circumferential margin involvement in rectal cancer resections has only been recognised relatively recently, ${ }^{15}{ }^{16}$ and the fact that the two best performing laboratories for reporting colonic cancer ranked fifth and eighth worst for reporting rectal cancer can be directly attributed to their low frequency of describing these margins.

Although $21.9 \%$ of reports of colonic cancer resections failed to fulfil all of the minimum criteria, table 3 shows that each of the five individual items of information required to meet this standard were generally well reported, with median values of $100 \%, 100 \%, 98.6 \%, 95.3 \%$, and $92.3 \%$, respectively. Apparently inadvertent omission of just one of the five items by pathologists who were otherwise diligent in recording the information seemed to be responsible for the failure of most of the reports. We believe that this is strong evidence for a need for a formal reporting process using structured template proformas which, by serving as a prompt for the pathologist, would ensure that a statement on each critical item of information was contained within the report. A number of such template proformas have been published $^{6} 89$ but informal discussions with pathologist colleagues suggests a reluctance to use them because they are perceived as being too detailed for routine use, including items of information of allegedly unconfirmed importance. Mindful of this, the histopathologists advising the Royal College of Surgeons of England/Association of Coloproctology on guidelines for the management of colorectal cancer, after wide consultation with other groups, have produced a minimum data template proforma for reporting resection specimens. ${ }^{10}$ We advocate its use to all laboratories, in the hope that it might become a national standard for minimum reporting that will be subject to regular review. We are not suggesting that proforma reporting should entirely replace the current practice of using free text in reports. Pathologists must be free to include whatever information they regard as useful and there will always be reports that require description of special or unusual features in specimens. However, we believe that template proformas will go a long way towards improving the quality and completeness of the information provided for patient management. It will also facilitate recording of data on computerised information systems and its retrieval for pathological input into audit, clinical trials and cancer registration.

Apart from the introduction of template proforma reporting, what other mechanisms might help to improve the current unsatisfactory position? We believe that continuing medical education can contribute by informing pathologists of relatively new approaches to dissecting and sampling resection specimens to obtain the maximum amount of information in an efficient way, ${ }^{15}{ }^{16}$ and in highlighting how clinical decisions on adjuvant therapy depend more than ever on careful pathological staging and evaluation of resection margins. Assessment of competence and understanding in these areas should also be seen to be an important element of the accreditation process for trainee pathologists. Managers should ensure that laboratories have the resources to examine and report specimens to high standards and to maintain these standards through internal audit ${ }^{17}$-mechanisms already exist for encouraging this through national laboratory accreditation. Finally, pathologists must be motivated by being continually informed and reminded of the importance of their work and its quality by their surgeon and oncologist colleagues who should ensure that pathologists are seen as key members of the multidisciplinary teams which will deliver effective cancer care for the next millenium. ${ }^{18}$ The recognition of individual consultants as lead pathologists for site-specific cancers within the developing cancer units and centres should help considerably in this respect.

We wish to thank all of the consultant pathologists and staff of the 17 histopathology laboratories in Wales for providing access to the pathology reports, and Rita Carter and Anne Whitlow who collected the data from individual hospitals. We also thank the Clinical Effectiveness Support Unit (Wales) for data the Clinical Effectiveness Support Unit (Wales) for data Health for financial support.

1 Hermanek P, Sobin LH. Colorectal carcinoma. In: Hermanek P, Gospodarowicz MK, Henson DE, Hutter RVP, Sobin LH, eds. Prognostic factors in cancer. Berlin: Springer, 1995:64-79.

2 Slevin ML. Adjuvant treatment for colorectal cancer: no more room for nihilism. $B M 7$ 1996:312:392-3.

3 Adam IJ, Mohamdee MO, Martin IG, Scott N, Finan PJ, Johnston $\mathrm{D}$, et al. Role of circumferential margin involvement in the local recurrence of rectal cancer. Lancet 1994; 344:707-11.

4 Krook JE, Moertel CG, Gunderson LL, Wieand HS, Collins RT, Beart RW, et al. Effective surgical adjuvant therapy for high-risk rectal carcinoma. $N$ Engl f Med 1991; 324:709-15.

5 Morson BC, Dawson IMP, Day DW, Jass JR, Price AB, Williams GT. Gastrointestinal pathology. 3rd edn. Oxford: Blackwell Scientific Publications, 1990.

6 Rosai J. Ackerman's Surgical pathology. 8th edn. St Louis: Mosby, 1996.

7 Williams NS, Jass JR, Dixon MF, Quirke P, Johnson RJ, Robinson PJ, et al. Handbook for the clinicopathological assessment and staging of colorectal cancer. London: UK assessment and staging of colorectal cancer. London:

8 Henson DE, Hutter RVP, Sobin LH, Bowman HE. Protocol for the examination of specimens removed from patients with colorectal carcinoma: a basis for checklists. Arch Pathol Lab Med 1994;118:122-5.

9 Association of Directors of Anatomic and Surgical Pathology. Recommendations for the reporting of resected large ogy. Recommendations for the reporting of resecte
intestinal carcinomas. Hum Pathol 1996;27:5-8.

10 Royal College of Surgeons of England/Association of Coloproctology. Guidelines for the management of colorectal cancer. London: Royal College of Pathologists of England, 1996.

11 Stamatakis JD. An independent audit of colerectal cancer in two UK health regions [abstract]. $B r \mathcal{F}$ Cancer 1996; 74(Suppl XXVIII): 50 .

$12 \mathrm{Ng}$ IOL, Luk ISC, Yuen ST, Lau PWK, Pritchett CJ, Ng M, et al. Surgical clearance in resected rectal carcinomas; a multivariate analysis of clinicopathologic features. Cancer 1993;71:1972-6.

13 Hermanek P, Sobin LH (eds). TNM classification of malignant tumors. 4th edn. Berlin: Springer Verlag, 1992.

14 Stamatakis JD. An independent audit of colorectal cancer in two UK health regions. [abstract]. $B r \mathcal{F}$ Cancer 1996;74 (Suppl xxviii): 5 .

15 Chan KW, Boey J, Wong SKC. A method for reporting radial invasion and surgical clearance of rectal cancer. Histopathology 1985;9:1319-27.

16 Quirke P, Durdey P, Dixon MF, Williams NS. Local recurQuirke $P$, Durdey P, Dixon MF, Williams NS. Local recur-
rence of rectal adenocarcinoma due to inadequate surgical resection: histopathological study of lateral tumour spread and surgical excision. Lancet 1986;ii:996-9.

17 Campbell F, Griffiths DFR. Quantitative audit of the contents of histopathology reports. $\mathcal{F}$ Clin Pathol 1994;47: $360-1$.

18 Department of Health. A policy framework for commisioning cancer services. London: HMSO, 1994. 
Colorectal cancer pathology reporting: a regional audit. A D Bull, $A$ H B Biffin, f Mella, $A G$ Radcliffe, $\mathcal{\exists} D$ Stamatakis, $R \mathcal{F} C$ Steele, $G T$ Williams. J Clin Pathol 1997;50:138-142.

There were several errors in the abstract to this article. The correct abstract is published below.

\begin{abstract}
Aims-To audit the information content of pathology reports of colorectal cancer specimens in one National Health Service region.

Methods-All reports of colorectal cancer resection specimens from the 17 NHS histopathology laboratories in Wales during 1993 were evaluated against: (a) standards previously agreed as desirable by pathologists in Wales;
\end{abstract}

and (b) standards considered to be the minimum required for informed patien management.

Results-1242 reports were audited. There was notable variation in the performance of different laboratories and in the completeness of reporting of individual items of information. While many items were generally well reported, only $51.5 \%$ of rectal cancer reports contained a statement on the completeness of excision at the circumferential resection margin and only $30 \%$ of all reports stated the number of involved lymph nodes. All of the previously agreed items were contained in only $11.3 \%$ of reports on colonic tumours and $4.0 \%$ of reports on rectal tumours. Seventy eight per cent of colonic carcinoma reports and $46.6 \%$ of rectal carcinoma reports met the minimum standards.
Conclusions-The informational content of many routine pathology reports on colorectal cancer resection specimens is inadequate for quality patient management, for ensuring a clinically effective cancer service through audit, and for cancer registration. Template proforma reporting using nationally agreed standards is recommended as a remedy for this, along with improved education, review of laboratory practices in the light of current knowledge, and further motivation of pathologists through their involvement in multidisciplinary cancer management teams.

In the same article reference 14 should have read: Jass JR, Love SB, Northover JMA. A new prognostic classification of rectal cancer. Lancet 1987;i:1303-6, and not as published. 